\title{
SOLUTION OF THE ASYMMETRIC MIXING PROBLEM*
}

\author{
DENISE KIRSCHNER ${ }^{\dagger}$ AND JEFF MORGAN ${ }^{\ddagger}$
}

\begin{abstract}
In this paper, we develop the framework for the use of the asymmetric mixing function that allows for a two-sex pair-formation model with self-selection, used in sexually transmitted diseases. We prove existence and uniqueness of a solution assuming the population distributions are known.
\end{abstract}

Key words. integral equations, fixed point method, monotonicity, mixing functions

AMS subject classifications. 92D30, 45A05

PII. S003613999427752X

1. Introduction. The procedure of who mixes with whom during a sexual encounter is a phenomenon which has certainly existed for centuries, but the question of how this occurs has only recently been posed (cf. $[1,2,6,7,9,12,13,16,22,24$, $25]$ ). One aspect of the information gained in the answering of this question is that it can assist epidemiologists in the study of sexually transmitted diseases.

Functions which describe this partner selection process have been developed based on a characteristic of each individual that would lead them to the pairing. The most common method of classification has been to put individuals in a class describing their number of different sexual partners per year. A person (whether male or female) is placed in the category of $r$ if their desired number of partners is $r(>0)$. We balance this with a term $r_{i}(t, r)$, which is the actual number of different partners one has, given that one is in category $r$ ( $i$ represents either male or female). Consequently, it is necessary to also define the populations where each of these individuals "live," namely, $N_{i}(t, r)$, the sexually active population of category $r$, of sex $i$, at time $t .{ }^{1}$ For disease modeling, this grouping can also aid in understanding the individual's risk of becoming infected with a sexually transmitted disease. This stems from the fact that data seems to indicate that the risk of becoming infected with a sexually transmitted disease is highly correlated with the number of different sexual partners one has [21, $26]$.

${ }^{*}$ Received by the editors November 23, 1994; accepted for publication (in revised form) November 17, 1996; published electronically March 24, 1998.

http://www.siam.org/journals/siap/58-3/27752.html

${ }^{\dagger}$ Department of Microbiology and Immunology, University of Michigan Medical School, 6730 Medical Science II, 1150 West Medical Center Drive, Ann Arbor, MI 48109-0620 (kirschne@umich.edu). This research was conducted while the author was at the Department of Mathematics, Texas A \& M University, College Station, TX 77843-3368. This research was supported in part by NSF grant DMS 9596037.

${ }^{\ddagger}$ Department of Mathematics, Texas A \& M University, College Station, TX 77843-3368 (morgan@math.tamu.edu). This research was supported in part by NSF grant 9208046.

${ }^{1}$ Traditionally, the function $r_{i}(t, r)$ depended only on the time and the class, $r$. However, it has been recently noted by Castillo-Chavez $[11,18]$ that this may not be completely correct. We generally assume that $r_{i}(t, r)>0$ for all $r$ regardless of whether or not the population $N_{i}(t, r)>0$. In the cases where $r$ is large, for example, and $N_{i}(t, r)=0$ or $N_{i}(t, r)$ is too small, it is more precise to have considered $r_{i}(t, r)$ as functions of not only $t$ and $r$, but also of the population sizes. To the end, consider the functions $M_{i}(t)=\int_{0}^{\infty} N_{i}(t, r) d r, i \in\{M, F\}$. Then, more appropriately, $r_{i}(t, r)$ is actually a function of $r_{i}\left(t, r, M_{i}(t), M_{j}(t)\right)$ for $i, j \in\{M, F\}, i \neq j$. In particular, $r_{i}\left(t, r, M_{i}(t), 0\right)=0$, $i \in\{M, F\}$. For this study, however, we assume the population sizes make sense, and require $r_{i}$ to be a function of $t$ and $r$ alone. 
For a purely heterosexual population, an S-I-A model has been developed to describe the spread of AIDS [11, 14, 19, 20] (see Appendix A). Two key ingredients of this model are the mixing functions $\rho_{F}$ and $\rho_{M}$, that describe the way in which people select partners. These functions are coupled to the S-I-A model through the nonlinear integral equations (3a)-(3b) (see section 2 below). As a result, an important first step in the process of determining the well-posedness of the S-I-A model is the solvability of the equations satisfied by $\rho_{F}$ and $\rho_{M}$ under the assumption that the functions $r_{i}$ and $N_{i}$ are known. The work at hand addresses this problem. Subsequent work will address the well-posedness of the S-I-A model.

To this end, section 2 introduces the mixing functions, and in section 3 we prove existence and uniqueness of a solution. In section 4 we discuss an example.

2. Mixing functions: $\boldsymbol{\rho}_{\boldsymbol{F}}(\boldsymbol{t}, \boldsymbol{r}, s)$ and $\boldsymbol{\rho}_{\boldsymbol{M}}(\boldsymbol{t}, \boldsymbol{r}, s)$. Define the functions $\rho_{i}(t, r, s)$ as the density functions for population $i$ of category $r$ of their partners of category $s$ of sex $j, i \neq j$. Many have explored these partner selections and pair formations in the setting of disease modeling, [5], [7], [8], [10], [15], [18], [23]. In 1991, Busenberg and Castillo-Chavez [4] gave nine general cases for mixing functions, each arising from different assumptions. Here we present a few to motivate our choice.

For any one- or two-sex model with heterogeneous activity Blythe and CastilloChavez [3] described mixing functions of the form $\rho_{i}(t, r, s), i \in\{M, F\}$. The idea is

that $\int_{s}^{s+\Delta s} \rho_{i}(t, r, x) d x$ represents the fraction of partners that a person with activity $r$ has among individuals with activities in the range $[s, s+\Delta s]$.

For these functions $\rho_{i}(t, r, s)$, there are some natural conditions which arise. Namely,

$$
\int_{0}^{\infty} \rho_{i}(t, r, s) d s=1, \quad \rho_{i}(t, r, s) \geq 0
$$

and

$$
\rho_{M}(t, r, s) r_{M}(t, r) N_{M}(t, r)=\rho_{F}(t, s, r) r_{F}(t, s) N_{F}(t, s),
$$

where $r_{i}(t, r) N_{i}(t, r)$ represents the distribution of the total sexually active population for each category $r$. Equations (1a) and (2a) clearly imply

$$
\int_{0}^{\infty} r_{M}(t, r) N_{M}(t, r) d r=\int_{0}^{\infty} r_{F}(t, s) N_{F}(t, s) d s .
$$

Equation (1a) guarantees that the probability density function integrates to 1, so that the people of category $r$ actually have an average of $r_{i}(t, r)$ different partners per year. Equation (2a) is a balancing equation stating that the number of partners with classification $s$ with whom people of class $r$ have sex is the same as the number of partners with classification $r$ with whom people of class $s$ have sex.

In a single-sex scenario, proportionate mixing is represented as (cf. [3], [10])

$$
\rho(s, r)=\frac{r N(r)}{\int_{0}^{\infty} x N(x) d x} .
$$

In [8], Busenberg and Castillo-Chavez present a scheme as well as discuss solvability for a two-sex mixing problem. They show that

$$
\rho_{M}(s)=\frac{r_{F}(s) N_{F}(s)}{\int_{0}^{\infty} r_{M}(x) N_{M}(x) d x} \text { and } \rho_{F}(r)=\frac{r_{M}(r) N_{M}(r)}{\int_{0}^{\infty} r_{F}(x) N_{F}(x) d x}
$$


are the only separable two-sex mixing functions satisfying conditions (1a,b) and (2a,b).

Hyman and Stanley [15] introduced a modified version of this mixing function, which is a more general alternative to proportionate mixing, and that differs from the equations of Castillo-Chavez and Busenberg through multiplication by a function $f(s, r)$. This function $f(s, r)$ is arbitrary and can aid in fine tuning the behavior of the mixing.

Expanding the notion of proportionate mixing, we refer to the heterosexual population self-selection rule from Stanley in [17], [23], namely, the asymmetric rule, where only one of the sexes in the pairing does the choosing. If we suppose the females do the choosing, then

$$
\begin{aligned}
\rho_{F}(t, r, s) & =\frac{\left[1-\int_{0}^{r} \rho_{M}(t, s, x) d x\right] f_{F}(r, s) r_{M}(t, s) N_{M}(t, s)}{\int_{0}^{\infty}\left(1-\int_{0}^{r} \rho_{M}(t, y, u) d u\right) f_{F}(r, y) r_{M}(t, y) N_{M}(t, y) d y}, \\
\rho_{M}(t, r, s) & =\frac{\rho_{F}(t, s, r) r_{F}(t, s) N_{F}(t, s)}{r_{M}(t, r) N_{M}(t, r)} .
\end{aligned}
$$

The idea is as follows. The numerator of the right-hand side of the first expression comes from multiplying the distribution of females and their male partners, times the acceptance function $f_{F}$ of male partners of class $s$ for the females of class $r$, times the actual number of male partners those females have, times the number of available males. This is normalized by integrating over all the possible male partners of class $s$. Notice that $(3 \mathrm{~b})$ is simply a restatement of $(2 \mathrm{a})$.

The remainder of this work focuses on the solution of the system $(1 \mathrm{a}, \mathrm{b})-(3 \mathrm{a}, \mathrm{b})$, the so-called asymmetric mixing problem.

3. Existence and uniqueness of a solution to the asymmetric mixing problem. For ease of calculation, we begin by manipulating the expression for the female infectivity, (3a), by applying condition (1) to replace $1-\int_{0}^{r} \rho_{M}(t, r, x) d x$ with $\int_{r}^{\infty} \rho_{M}(t, r, x) d x$. Then, substituting equation (3b) into (3a) yields an integral equation for $\rho_{F}(t, r, s)$, namely,

$$
\rho_{F}(t, r, s)=\frac{\int_{r}^{\infty} \frac{\rho_{F}(t, x, s) r_{F}(t, x) N_{F}(t, x) d x}{r_{M}(t, s) N_{M}(t, s)} \cdot f_{F}(r, s) r_{M}(t, s) N_{M}(t, s)}{\int_{0}^{\infty} \int_{r}^{\infty} \frac{\rho_{F}(t, u, y) r_{F}(t, u) N_{F}(t, u) d u}{r_{M}(t, y) N_{M}(t, y)} f_{F}(r, y) r_{M}(t, y) N_{M}(t, y) d y} .
$$

Canceling like terms and suppressing the time notation (since we require this to hold for all time) yields

$$
\rho_{F}(r, s)=\frac{\int_{r}^{\infty} \rho_{F}(x, s) r_{F}(x) N_{F}(x) d x f_{F}(r, s)}{\int_{0}^{\infty}\left[\int_{r}^{\infty} \rho_{F}(x, y) r_{F}(x) N_{F}(x) d x\right] f_{F}(r, y) d y} .
$$

Define

$$
w_{i}(x)=r_{i}(x) N_{i}(x) \quad \text { for } i=\{F, M\} .
$$

Then the integral equation (4) becomes

$$
\rho_{F}(r, s)=\frac{\int_{r}^{\infty} \rho_{F}(x, s) w_{F}(x) d x f_{F}(r, s)}{\int_{0}^{\infty}\left[\int_{r}^{\infty} \rho_{F}(x, y) w_{F}(x) d x\right] f_{F}(r, y) d y} .
$$


If we apply equation (1a), namely, $\int_{0}^{\infty} \rho_{M}(r, s) d s=1$, then substituting (3b) for $\rho_{M}(r, s)$ yields

$$
\int_{0}^{\infty} \frac{\rho_{F}(s, r) w_{F}(s)}{w_{M}(r)} d s=1 .
$$

Hence,

$$
w_{M}(s)=\int_{0}^{\infty} \rho_{F}(x, s) w_{F}(x) d x .
$$

It is the solution to the integral equation (5) together with condition (6) that is explored below. ${ }^{2}$

Remark. Since $\rho_{M}(r, s)$ and $f_{M}(r, s)$ do not appear in (5)-(6), we will simply write $\rho(r, s)$ and $f(r, s)$ to denote $\rho_{F}(r, s)$ and $f_{F}(r, s)$, respectively.

In order to obtain a solution to (5)-(6), it is necessary to impose some reasonable assumptions on $f, w_{F}$, and $w_{M}$.

Assumptions.

(Ai) $w_{M}, w_{F} \in C^{1}\left(\mathrm{R}_{+}\right) \cap L^{1}\left(\mathrm{R}_{+}\right)$,

(Aii) $f \in C^{1}\left(\mathrm{R}_{+}^{2}\right) \cap L^{\infty}\left(\mathrm{R}_{+}^{2}\right)$,

(Aiii) $w_{M}, w_{F}, f>0$,

(Aiv) $\int_{0}^{\infty} w_{M}(\tau) d \tau=\int_{0}^{\infty} w_{F}(\tau) d \tau$,

(Av) $\lim _{s \rightarrow \infty} w_{M}(s)=0$,

(Avi) For every $s>0$ there exists $\varepsilon_{s}>0$ such that $f(r, s) \geq \varepsilon_{s}$ for all $r>0$.

Conditions (Ai), (Aii), (Aiii), (Av) are not unreasonable, as choices for the functions $w_{M}, w_{F}$, and $f$ should satisfy these assumptions. Assumption (Aiv) is simply a restatement of (2b), and assumption (Avi) is technical in nature and needed for the analysis.

Multiply (5) by $w_{F}(r)$ and define $\mathcal{U}(r, s)=\int_{r}^{\infty} \rho(x, s) w_{F}(x) d x$. Then for each fixed $s$, we have a first-order differential equation in $\mathcal{U}$ with respect to $r$. Since (6) yields initial data for this differential equation, solving it yields the nonlinear integral equation

$$
\mathcal{U}(r, s)=w_{M}(s) \cdot \exp \left(-\int_{0}^{r} \frac{f(y, s) w_{F}(y)}{\int_{0}^{\infty} \mathcal{U}(y, t) f(y, t) d t} d y\right) .
$$

Symbolically, define $X=\left\{\mathcal{U} \in C\left(\mathrm{R}_{+}^{2}\right) \mid \mathcal{U}>0\right\}$ and

$$
T(\mathcal{U})(r, s)=w_{M}(s) \cdot \exp \left(-\int_{0}^{r} \frac{f(y, s) w_{F}(y)}{\int_{0}^{\infty} \mathcal{U}(y, t) f(y, t) d t} d y\right)
$$

for each $\mathcal{U} \in X$.

Note that the conditions on $w_{M}, w_{F}$, and $f$ guarantee that $T$ is well defined. In addition, the following lemma is a simple consequence of (8) and our assumptions.

LEMma 1. If $u_{1}, u_{2} \in X$ with $u_{1} \geq u_{2}$ then $T\left(u_{1}\right) \geq T\left(u_{2}\right)$. Furthermore, if $u \in X$ then

$$
0<T(u)(r, s) \leq w_{M}(s)
$$

\footnotetext{
${ }^{2}$ It should be clear that the solution to this problem is equivalent to solving the asymmetric mixing problem $(1 \mathrm{a}, \mathrm{b})-(3 \mathrm{a}, \mathrm{b})$.
} 
for all $(r, s) \in R_{+}^{2}$.

We can also obtain the following result.

Lemma 2. $\operatorname{Let}^{0}(r, s)=w_{M}(s)$ and define

$$
\mathcal{U}^{k}(r, s)=T\left(\mathcal{U}^{k-1}\right)(r, s)
$$

for each $k=1,2, \ldots$ Then

$$
0<\mathcal{U}^{k} \leq \mathcal{U}^{k-1} \leq \cdots \leq \mathcal{U}^{1} \leq \mathcal{U}^{0}=w_{M} .
$$

Furthermore, if we define

$$
V(r, s)=w_{M}(s) \exp \left(-\int_{0}^{r} \frac{2 f(y, s) w_{F}(y)}{\Psi(y)\left[\int_{0}^{\infty} w_{M}(\tau) d \tau-\int_{0}^{r} w_{F}(\tau) d \tau\right]} d y\right),
$$

then

$$
0<V \leq \mathcal{U}^{k} \leq \cdots \leq \mathcal{U}^{1} \leq \mathcal{U}^{0}=w_{M}
$$

Proof. Define a sequence $\left\{\mathcal{U}^{i}\right\}_{i=0}^{\infty} \subseteq X$ as follows. Let $\mathcal{U}^{0}(r, s)=w_{M}(s)$ and define $\mathcal{U}^{k}$ recursively by $\mathcal{U}^{k}(r, s)=T\left(\mathcal{U}^{k-1}\right)(r, s), k=\{1,2, \ldots\}$. It follows from Lemma 1 (above) that the $\mathcal{U}^{k}(r, s)$ are nested as $0<\mathcal{U}^{k} \leq \mathcal{U}^{k-1} \leq \cdots \leq \mathcal{U}^{1} \leq \mathcal{U}^{0} \leq w_{M}(s)$. Also, from the definition of $T$, we have

$$
\mathcal{U}^{k}(r, s)=w_{M}(s) \cdot \exp \left(-\int_{0}^{r} \frac{f(y, s) w_{F}(y)}{\int_{0}^{\infty} \mathcal{U}^{k-1}(y, t) f(y, t) d t} d y\right) .
$$

Using the fundamental theorem of calculus, we differentiate to obtain

$$
\mathcal{U}_{r}^{k}(r, s)=\mathcal{U}^{k}(r, s)\left(\frac{-f(r, s) w_{F}(r)}{\int_{0}^{\infty} \mathcal{U}^{k-1}(r, t) f(r, t) d t}\right) .
$$

Replacing $\mathcal{U}^{k}(r, s)$ by $\mathcal{U}^{k-1}(r, s)$ on the right-hand side and integrating both sides with respect to $s$ yields

$$
\int_{0}^{\infty} \frac{\mathcal{U}_{r}^{k}(r, s)}{w_{F}(r)} d s \geq-1, \quad \text { or equivalently, } \quad \frac{d}{d r} \int_{0}^{\infty} \mathcal{U}^{k}(r, s) d s \geq-w_{F}(r) .
$$

If both sides are integrated with respect to $r$, the expression becomes

$$
\int_{0}^{\infty} \mathcal{U}^{k}(r, s) d s \geq \int_{0}^{\infty} w_{M}(s) d s-\int_{0}^{r} w_{F}(\tau) d \tau
$$

Then $0<\mathcal{U}^{k}(r, s) \leq w_{M}(s)$ implies $0<\int_{a}^{b} \mathcal{U}^{k}(r, s) d s \leq \int_{a}^{b} w_{M}(s) d s$ for all $0 \leq a<b$. Furthermore, following directly from (Aiv), there must exist a continuous, positive, increasing function $g(r)$ satisfying $0<g(r)<\infty$, such that

$$
\int_{g(r)}^{\infty} w_{M}(\tau) d \tau \leq \frac{1}{2}\left[\int_{0}^{\infty} w_{M}(\tau) d \tau-\int_{0}^{r} w_{F}(\tau) d \tau\right]
$$


Combining these observations with (9) yields

$$
\frac{1}{2}\left[\int_{0}^{\infty} w_{M}(\tau) d \tau-\int_{0}^{r} w_{F}(\tau) d \tau\right] \leq \int_{0}^{g(r)} \mathcal{U}^{k}(r, s) d s,
$$

since from (9) we have

$$
\int_{0}^{g(r)} \mathcal{U}^{k}(r, s) d s+\int_{g(r)}^{\infty} \mathcal{U}^{k}(r, s) d s \geq \int_{0}^{\infty} w_{M}(\tau) d \tau-\int_{0}^{r} w_{F}(\tau) d \tau
$$

This implies

$$
\begin{aligned}
\int_{0}^{g(r)} \mathcal{U}^{k}(r, s) d s & \geq \int_{0}^{\infty} w_{M}(\tau) d \tau-\int_{0}^{r} w_{F}(\tau) d \tau-\int_{g(r)}^{\infty} \mathcal{U}^{k}(r, s) d s \\
& \geq \int_{0}^{\infty} w_{M}(\tau) d \tau-\int_{0}^{r} w_{F}(\tau) d \tau-\frac{1}{2}\left[\int_{0}^{\infty} w_{M}(\tau) d \tau-\int_{0}^{r} w_{F}(\tau) d \tau\right] \\
& =\frac{1}{2}\left[\int_{0}^{\infty} w_{M}(\tau) d \tau-\int_{0}^{r} w_{F}(\tau) d \tau\right] .
\end{aligned}
$$

Define $\Psi(y)=\min _{0 \leq t \leq g(y)} f(y, t)$. Then for $R>0$, there exists an $\varepsilon_{R}>0$ such that $\Psi(r) \geq \varepsilon_{R}$ for all $0 \leq r \leq R$. If we examine

$$
\mathcal{U}^{k+1}(r, s)=w_{M}(s) \cdot \exp \left(-\int_{0}^{r} \frac{f(y, s) w_{F}(y)}{\int_{0}^{\infty} \mathcal{U}^{k}(y, t) f(y, t) d t} d y\right),
$$

then our observations above imply

$$
\begin{aligned}
\mathcal{U}^{k+1}(r, s) & \geq w_{M}(s) \cdot \exp \left(-\int_{0}^{r} \frac{f(y, s) w_{F}(y)}{\int_{0}^{g(y)} \mathcal{U}^{k}(y, t) f(y, t) d t} d y\right) \\
& \geq w_{M}(s) \cdot \exp \left(-\int_{0}^{r} \frac{f(y, s) w_{F}(y)}{\Psi(y) \int_{0}^{g(y)} \mathcal{U}^{k}(y, t) d t} d y\right) \\
& \geq w_{M}(s) \cdot \exp \left(-\int_{0}^{r} \frac{f(y, s) w_{F}(y)}{\Psi(y) \frac{1}{2}\left[\int_{0}^{\infty} w_{M}(\tau) d \tau-\int_{0}^{r} w_{F}(\tau) d \tau\right]} d y\right)
\end{aligned}
$$

This bounds the sequence of $\mathcal{U}^{k}(r, s),\{k=1,2, \ldots\}$ away from zero. Namely, we have

$$
0<V(r, s) \leq \mathcal{U}^{k}(r, s) \leq \mathcal{U}^{k-1}(r, s) \leq \cdots \leq \mathcal{U}^{1}(r, s) \leq \mathcal{U}^{0}(r, s) \leq w_{M}(s),
$$

where

$$
V(r, s) \equiv w_{M}(s) \cdot \exp \left(-\int_{0}^{r} \frac{f(y, s) w_{F}(y)}{\Psi(y)\left[\frac{1}{2} \int_{0}^{\infty} w_{M}(\tau) d \tau-\int_{0}^{r} w_{F}(\tau) d \tau\right]} d y\right)
$$

is clearly a positive continuous function on $\mathrm{R}_{+}^{2}$.

Lemma 3. Fix $R>0 .\left\{\mathcal{U}^{k}\right\}$ is a convergent sequence in $B C([0, R] \times[0, \infty))$.

Proof. If $\mathcal{S}>0$ and we define $W=[0, R] \times[0, \mathcal{S}]$, then there exists an $\varepsilon_{R, \mathcal{S}}>0$ such that $0<\varepsilon_{R, \mathcal{S}} \leq V(r, s) \leq w_{M}(s)$ for all $r, s \in W$. Consequently, it follows from 
(8), (10) that $\left\{\mathcal{U}^{k}\right\}$ is bounded in $C^{1}(W)$. Now, let $\mathcal{S}_{1}>0$ such that $w_{M}(s) \leq 1$ for all $s \geq \mathcal{S}_{1}$. Define $W_{1}=[0, R] \times\left[0, \mathcal{S}_{1}\right]$. Then, there is a subsequence $\left\{\mathcal{U}^{k_{1, j}}\right\}_{j=1}^{\infty}$ of $\left\{\mathcal{U}^{k}\right\}$ which is Cauchy in $C\left(W_{1}\right)$. Similarly, there is an $\mathcal{S}_{2}>0$ such that $w_{M}(s) \leq \frac{1}{2}$ for all $s \geq \mathcal{S}_{2} \geq \mathcal{S}_{1}$, and there is a subsequence $\left\{\mathcal{U}^{k_{2, j}}\right\}_{j=1}^{\infty}$ of $\left\{\mathcal{U}^{k_{1, j}}\right\}_{j=1}^{\infty}$ which is Cauchy in $C\left(W_{2}\right)\left(W_{2}=[0, R] \times\left[0, \mathcal{S}_{2}\right]\right)$. Inductively, there exists an $\mathcal{S}_{n+1} \geq \mathcal{S}_{n}$ such that $w_{M}(s) \leq \frac{1}{n+1}$ for all $s \geq \mathcal{S}_{n+1}$, and there is a subsequence $\left\{\mathcal{U}^{k_{n+1, j}}\right\}_{j=1}^{\infty}$ of $\left\{\mathcal{U}^{k_{n, j}}\right\}_{j=1}^{\infty}$ which is Cauchy in $C\left(W_{n+1}\right)\left(W_{n+1}=[0, R] \times\left[0, \mathcal{S}_{n+1}\right]\right)$. Define $\hat{\mathcal{U}}^{j}=\mathcal{U}^{k_{j, j}}$ for $j=1,2, \ldots$ We will show that $\left\{\hat{\mathcal{U}}^{j}\right\}_{j=1}^{\infty}$ is a Cauchy sequence in $B C([0, R] \times[0, \infty))$.

To this end, let $\varepsilon>0$. There exists an $n \in \mathcal{N}$ such that $\frac{1}{n}<\varepsilon$. Then, $w_{M}(s) \leq \frac{1}{n}$ for all $s \geq \mathcal{S}_{n}$. Since $\left\{\mathcal{U}^{k_{n, i}}\right\}_{i=1}^{\infty}$ is Cauchy in $C\left(W_{n}\right)$, there exists a $K>0$ such that if $i, l>K$ then $\left\|\mathcal{U}^{k_{n, i}}-\mathcal{U}^{k_{n, l}}\right\|_{\infty, W_{n}}<\varepsilon$. Let $L=K+n$, and suppose $i, l \geq L$. Examine

$$
\left\|\hat{\mathcal{U}}^{i}-\hat{\mathcal{U}}^{l}\right\|_{\infty, W_{n}}=\left\|\mathcal{U}^{k_{i, i}}-\mathcal{U}^{k_{l, l}}\right\|_{\infty, W_{n}}
$$

Since $i$ and $l$ are larger than both $n$ and $K$, there exists $j_{i} \geq i$ and $j_{l} \geq l$ such that $\mathcal{U}^{k_{i, i}}=\mathcal{U}^{k_{n, j_{i}}}$ and $\mathcal{U}^{k_{l, l}}=\mathcal{U}^{k_{n, j_{l}}}$. Hence, $\left\|\hat{\mathcal{U}}^{i}-\hat{\mathcal{U}}^{l}\right\|_{\infty, W_{n}}<\varepsilon$. If we compare these sequences on $W^{n}=[0, R] \times\left[\mathcal{S}_{n}, \infty\right)$, then we have

$$
\left\|\hat{\mathcal{U}}^{i}-\hat{\mathcal{U}}^{l}\right\|_{\infty, W_{n}} \leq\left\|w_{M}\right\|_{\infty, W_{n}} \leq \frac{1}{n} \leq \varepsilon .
$$

Hence, $\left\{\hat{\mathcal{U}}^{i}\right\}_{i=1}^{\infty}$ is Cauchy in $B C([0, R] \times[0, \infty))$.

Consequently, there exists a $\mathcal{U} \in B C([0, R] \times[0, \infty))$ such that $\left\{\hat{\mathcal{U}}^{i}\right\}$ converges to $\mathcal{U}$ uniformly.

Now recall $\hat{\mathcal{U}}^{i}=\mathcal{U}^{k_{i, i}}$ for all $i$. From the convergence of this subsequence to $\mathcal{U}$ and the monotonicity proved in Lemma 2 , we have for all $m$,

$$
0<V(r, s) \leq \mathcal{U}(r, s) \leq \mathcal{U}^{m}(r, s) \leq \mathcal{U}^{m-1}(r, s) \leq \cdots \leq \mathcal{U}^{1}(r, s) .
$$

Thus, the monotonicity of this sequence and the uniform convergence of the subsequence $\left\{\mathcal{U}^{k_{i, i}}\right\}_{i=1}^{\infty}$ to $\mathcal{U}$ in $B C([0, R] \times[0, \infty))$ yield the uniform convergence of $\left\{\mathcal{U}^{i}\right\}_{i=1}^{\infty}$ to $\mathcal{U}$ in $B C([0, R] \times[0, \infty))$. Note that since $R>0$ was arbitrary, we have actually proved that there exists $\mathcal{U} \in X$ such that $\left\{\mathcal{U}^{i}\right\}_{i=1}^{\infty}$ converges uniformly to $\mathcal{U}$ on $B C([0, R] \times[0, \infty))$ for all $R>0$.

Proposition 1. Under assumptions $(\mathrm{Ai})-(\mathrm{Av})$, there exists a unique, positive, continuous solution $\mathcal{U} \in X$ of (7). Furthermore, the solution satisfies

$$
0<\mathcal{U}(r, s)<w_{M}(s) \text { for }(r, s) \in \mathrm{R}_{+}^{2}
$$

and

$$
\int_{0}^{\infty} \mathcal{U}(r, s) d s=\int_{0}^{\infty} w_{M}(s) d s-\int_{0}^{r} w_{F}(s) d s \text { for } r>0
$$

Proof. Let $\varepsilon>0$ and suppose $\mathcal{U}$ is given as in the proof of Lemma 3. Consider

$$
\left\|T\left(\mathcal{U}^{k}\right)-T(\mathcal{U})\right\|_{\infty, W_{\infty}} \leq\left\|T\left(\mathcal{U}^{k}\right)-T(\mathcal{U})\right\|_{\infty, W_{n}}+\left\|T\left(\mathcal{U}^{k}\right)-T(\mathcal{U})\right\|_{\infty, W_{\mathcal{S}_{n}}},
$$

where $W_{\mathcal{S}_{n \infty}}=[0, R] \times\left[S_{n}, \infty\right)$ and $W_{\infty}=[0, R] \times[0, \infty)$. Let $\varepsilon>0$ and choose $n$ such that if $s \geq S_{n}$, then $w_{M}(s)<\varepsilon$. This implies that the second term in the right-hand side of (13) is bounded by $\varepsilon$ for $k$ sufficiently large. 
It remains to show that the first term on the right-hand side of (13) can be made arbitrarily small by taking $k$ sufficiently large. To this end, it is sufficient to prove that there exists a $K \in N$ such that if $k \geq K$ then

$$
\left|\int_{0}^{R} \frac{w_{F}(y) d y}{\int_{0}^{\infty} \mathcal{U}^{k}(y, t) f(y, t) d t}-\int_{0}^{R} \frac{w_{F}(y) d y}{\int_{0}^{\infty} \mathcal{U}(y, t) f(y, t) d t}\right|<\varepsilon .
$$

But,

$$
\begin{aligned}
& \left|\int_{0}^{R} \frac{w_{F}(y) d y}{\int_{0}^{\infty} \mathcal{U}^{k}(y, t) f(y, t) d t}-\int_{0}^{R} \frac{w_{F}(y) d y}{\int_{0}^{\infty} \mathcal{U}(y, t) f(y, t) d t}\right| \\
= & \left|\int_{0}^{R} \frac{w_{F}(y) \int_{0}^{\infty}\left(\mathcal{U}^{k}(y, t)-\mathcal{U}(y, t)\right) f(y, t) d t d y}{\int_{0}^{\infty} \mathcal{U}^{k}(y, t) f(y, t) d t \int_{0}^{\infty} \mathcal{U}(y, t) f(y, t) d t}\right| \\
\leq & \left\|\mathcal{U}^{k}-\mathcal{U}\right\|_{\infty, W_{\infty}}\|f\|_{\infty, W_{\infty}} \int_{0}^{R} \frac{w_{F}(y) d y}{\left(\int_{0}^{\infty} \mathcal{U}(y, t) f(y, t) d t\right)^{2}} \\
\leq & \frac{\left\|\mathcal{U}^{k}-\mathcal{U}\right\|_{\infty, W_{\infty}}\|f\|_{\infty, W_{\infty}}\left\|w_{F}\right\|_{1,[0, R]}}{\delta_{R}},
\end{aligned}
$$

where $0<\delta_{R}=\inf _{0 \leq y \leq R}\left(\int_{0}^{\infty} \mathcal{U}(y, t) f(y, t) d t\right)^{2}$. Since $\left\{\mathcal{U}^{k}\right\}_{k=1}^{\infty}$ converges uniformly to $\mathcal{U}$ in $B C\left(W_{\infty}\right)$, we must have $T(\mathcal{U})=\mathcal{U}$.

Consequently, $\mathcal{U}$ solves $(7)$. Let's prove that there are no other positive continuous solutions. Since $\mathcal{U}=T(\mathcal{U})$ we have

$$
\mathcal{U}_{r}(r, s)=\mathcal{U}(r, s)\left(\frac{-f(r, s) w_{F}(r)}{\int_{0}^{\infty} \mathcal{U}(r, t) f(r, t) d t}\right) .
$$

If we integrate with respect to $s$ then

$$
\int_{0}^{\infty} \frac{\mathcal{U}_{r}(r, s)}{w_{F}(s)} d s=-1
$$

That is,

$$
\frac{d}{d r} \int_{0}^{\infty} \mathcal{U}(r, s) d s=-w_{F}(r)
$$

Therefore, integration with respect to $r$ yields

$$
\int_{0}^{\infty} \mathcal{U}(r, s) d s=\int_{0}^{\infty} w_{M}(s) d s-\int_{0}^{r} w_{F}(\tau) d \tau .
$$

Now assume that there exists another positive continuous solution $V$ of (11). Then $V=T^{k}(V) \leq T^{k}\left(w_{M}\right)=\mathcal{U}^{k}$ for all $r, s$, which implies $V \leq \mathcal{U}$ for all $r, s$. On the other hand, since $V=T(V)$, we have

$$
\int_{0}^{\infty} V(r, s) d s=\int_{0}^{\infty} w_{M}(s) d s-\int_{0}^{r} w_{F}(\tau) d \tau,
$$

as above. Therefore $\int_{0}^{\infty} \mathcal{U}(r, s) d s=\int_{0}^{\infty} V(r, s) d s$. Consequently, we must have $\mathcal{U}=$ $V$. 
To complete our analysis of (5)-(6) it remains to prove the following result.

THEOREM 1. If (Ai)-(Avi) hold then there exists a unique positive continuous solution of (5)-(6).

Proof. To begin, let $\mathcal{U}(r, s)$ be the unique solution of (7). Using the fundamental theorem of calculus, differentiate both sides with respect to $r$, yielding

$$
\mathcal{U}_{r}(r, s)=\mathcal{U}(r, s)\left(\frac{-f(r, s) w_{F}(r)}{\int_{0}^{\infty} \mathcal{U}(r, t) f(r, t) d t}\right),
$$

or equivalently,

$$
\frac{\mathcal{U}_{r}(r, s)}{-w_{F}(r)}=\frac{\mathcal{U}(r, s) f(r, s)}{\int_{0}^{\infty} \mathcal{U}(r, t) f(r, t) d t} .
$$

Now define

$$
\rho(r, s)=-\frac{\mathcal{U}_{r}(r, s)}{w_{F}(r)} \text { for }(r, s) \in \mathrm{R}_{+}^{2} .
$$

Note that $\rho$ is positive and continuous. To show that $\rho$ satisfies (5)-(6) we need

$$
\int_{r}^{\infty} \rho(x, s) w_{F}(x) d x=\mathcal{U}(r, s)
$$

and

$$
w_{M}(s)=\int_{0}^{\infty} \rho(x, s) w_{F}(x) d x .
$$

For (19), note that by definition

$$
\begin{aligned}
\int_{0}^{\infty} \rho(x, s) w_{F}(x) d x & =\int_{0}^{\infty}-\mathcal{U}_{r}(r, s) d r \\
& =\mathcal{U}(0, s)-\operatorname{Lim}_{R \rightarrow \infty} \mathcal{U}(R, s) \\
& =w_{M}(s)-\operatorname{Lim}_{R \rightarrow \infty} \mathcal{U}(R, s) .
\end{aligned}
$$

Also, for (18), we have by definition,

$$
\begin{aligned}
\int_{r}^{\infty} \rho(x, s) w_{F}(x) d x & =\int_{r}^{\infty}-\mathcal{U}_{r}(r, s) d x \\
& =\mathcal{U}(r, s)-\operatorname{Lim}_{R \rightarrow \infty} \mathcal{U}(R, s) .
\end{aligned}
$$

So, both (18) and (19) will hold provided $\operatorname{Lim}_{R \rightarrow \infty} \mathcal{U}(R, s)=0$ for $s>0$, fixed.

But, for $s>0$ fixed,

$$
\mathcal{U}(r, s)=w_{M}(s) \cdot \exp \left(-\int_{0}^{r} \frac{f(y, s) w_{F}(y)}{\int_{0}^{\infty} \mathcal{U}(y, t) f(y, t) d t} d y\right)
$$


So,

$$
\begin{aligned}
0<\mathcal{U}(r, s) & \leq w_{M}(s) \cdot \exp \left(-\int_{0}^{r} \frac{f(y, s) w_{F}(y)}{\|f\|_{\infty} \int_{0}^{\infty} \mathcal{U}(y, t) d t} d y\right) \\
& \leq w_{M}(s) \cdot \exp \left(\frac{-1}{\|f\|_{\infty}} \int_{0}^{r} \frac{f(y, s) w_{F}(y)}{\int_{0}^{\infty} w_{M}(\tau) d \tau-\int_{0}^{y} w_{F}(\tau) d \tau} d y\right)(\text { from (11)) } \\
& \leq w_{M}(s) \cdot \exp \left(\frac{-\varepsilon_{s}}{\|f\|_{\infty}} \int_{0}^{r} \frac{w(y)}{\int_{0}^{\infty} w_{M}(\tau) d \tau-\int_{0}^{y} w_{F}(\tau) d \tau} d y\right)(\text { from (Avi)) } \\
& \leq w_{M}(s) \cdot \exp \left(\frac { \varepsilon _ { s } } { \| f \| _ { \infty } } \left[\ln \left|\int_{0}^{\infty} w_{M}(\tau) d \tau-\int_{0}^{r} w(\tau) d \tau\right|\right.\right. \\
& \left.\left.\quad-\ln \left(\int_{0}^{\infty} w_{M}(\tau) d \tau\right)\right]\right)
\end{aligned}
$$

after integration. Recall from (Aiv) that

$$
\int_{0}^{\infty} w_{M}(\tau) d \tau=\int_{0}^{\infty} w_{F}(\tau) d \tau
$$

Hence, $\lim _{r \rightarrow \infty} \mathcal{U}(r, s)=0$.

Therefore, we have existence of a solution of (5)-(6). For uniqueness, note that if $\tilde{\rho}$ is also a positive, continuous solution of $(5)-(6)$ then $\tilde{\mathcal{U}}(r, s) \equiv w_{M}(s)-$ $\int_{0}^{r} \tilde{\rho}(x, s) w_{F}(x) d x$ solves $(7)$, as does $\mathcal{U}(r, s)=w_{M}(s)-\int_{0}^{r} \rho(x, s) w_{F}(x) d x$. But (7) has a unique solution, which implies $\mathcal{U}=\tilde{\mathcal{U}}$, and hence $\int_{0}^{r} \rho(x, s) w_{F}(x) d x=$ $\int_{0}^{r} \tilde{\rho}(x, s) w_{F}(x) d x$. As a result, $\rho=\tilde{\rho}$.

4. Comments and an example. We want to first point out that the method we used is constructive, lending itself to numerical interpretation and exploration.

For an example, consider a choice of an acceptance function which is the density function of a Gaussian-like distribution

$$
f(r, s)=c \cdot \exp \left\{-\frac{1}{2 \varepsilon^{2}}\left(\frac{r-s}{r+a}\right)^{2}\right\}, \quad r, s \geq 0,
$$

where $c, a$, and $\varepsilon$ are positive constants. Here, the variable $s$ represents the class of the partner and $r$ is the fixed class of the person. These assumptions allow for a widely spread out distribution for high risk category individuals and a very narrow distribution for low risk category individuals. The $(r+a)$ prevents the low risk category distribution from degenerating (i.e., there is some minimum variance). The parameter $a$ might be of order 1 . So this choice of $f$ allows the distinction between high and low risk class groups (i.e., class factors of $r=3$ or $r=4$ give basically the same distribution, but one very different from that of $r=15$ ).

We assume the population distributions are given by $N_{i}(x)=\frac{C_{i}}{\left(x+b_{i}\right)^{4}}$ for $i \in$ $\{M, F\}$ and $C_{i}, b_{i}, i \in\{M, F\}$ are positive constants [14]. This assumes that there is a large distribution of individuals in a low risk class and a small number in the high risk classes. For the class distributions, we assume $r_{i}(x)=x+b_{i}, i \in\{M, F\}$. This would describe a population where the desired number of partners per year equals the actual number, plus possibly a correcting term relating to the available population. Hence, the probability distributions, $w_{i}(x)=r_{i}(x) N_{i}(x)$, are $w_{i}(x)=\frac{C_{i}}{\left(x+b_{i}\right)^{3}}, i \in\{M, F\}$. 
Note that the assumptions (Ai)-(Avi) are easily satisfied by these choices of functions $w_{i}(x), f(r, s)$.

Finally, Hyman and Stanley have numerically explored this and other mixing functions with different acceptance functions. They present numerical results for the full system (20)-(23) given in Appendix A coupled with appropriate initial conditions and the nonlinear integral equation for $\rho_{i}(t, r, s)$, and they give numerical estimates for special cases of the asymmetric mixing functions, $\rho_{i}(t, r, s), i \in\{M, F\}$ [17].

Appendix A. For a purely heterosexual population, the following model has been developed to describe the spread of AIDS [11, 14, 19, 20]. Let $S_{i}(t, r)$ denote the susceptible population at time $t$ with risk $r$ and sex $i$; let $I_{i}(t, \tau, r)$ denote the infected population at time $t$, time since infection $\tau$, with risk $r$ and sex $i$; let $A_{i}(t, \tau, r)$ denote the AIDS population (showing clinical AIDS symptoms) at time $t$, time since conversion $\hat{\tau}$, with risk $r$ and sex $i$. The interaction of all three populations has the form

$$
\begin{aligned}
& \partial S_{i}(t, r) / \partial t=\mu\left(S o_{i}(r)-S_{i}(t, r)\right)-\lambda_{i}(t, r) S_{i}(t, r), \\
& I_{i}(t, r, 0)=\lambda_{i}(t, r) S_{i}(t, r), \\
& \partial I_{i}(t, r, \tau) / \partial t+\partial I_{i}(t, r, \tau) / \partial \tau=-(\gamma(\tau)+\mu) I_{i}(t, r, \tau), \\
& A_{i}(t, r, 0)=\int_{0}^{\infty} \gamma(\tau) I_{i}(t, r, \tau) d \tau, \\
& \partial A_{i}(t, r, \hat{\tau}) / \partial t+\partial A_{i}(t, r, \hat{\tau}) / \partial \hat{\tau}=-(\delta(\hat{\tau})+\mu) A_{i}(t, r, \hat{\tau}), \\
& \lambda_{i}(t, r)=r_{i}(t, r) \int_{0}^{\infty} \rho_{i}(t, r, s) K_{j}(t, r, s) d s, \quad i \neq j,
\end{aligned}
$$

where

$$
K_{j}(t, r, s)=\int_{0}^{\infty} \beta(\tau, r) \frac{I_{j}(t, s, \tau)}{N_{j}(t, s)} d \tau \quad \text { and } \quad N_{j}(t, s)=S_{j}(t, s)+\int_{0}^{\infty} I_{j}(t, \tau, s) d \tau .
$$

Here $i, j \in\{M, F\}, \mu$ and $\delta(\hat{\tau})$ are death rates, and $\lambda_{i}(t, r)$ and $\gamma(\tau)$ are the infection and conversion rates, respectively. $S o_{i}$ is the steady state population in the absence of AIDS; $K_{j}(t, r, s)$ is the probability that a person of sex $j$ and risk $r$ will be infected by a person of sex $i$ with risk $s ; r_{i}(t, r)$ is the actual number of different partners per year; and $\rho_{i}(t, r, s)$ is a mixing function (discussed in section 2) which satisfies a nonlinear integral equation. The system (20)-(23) above is coupled with nonnegative initial data $S_{i}(0, r), I_{i}(0, r, \tau)$, and $A_{i}(0, r, \tau)$.

\section{REFERENCES}

[1] R. M. Anderson (1989), Editorial review on mathematical and statistical studies of the epidemiology of HIV, AIDS, 3, pp. 333-346.

[2] R. M. Anderson and R. M. May (1987), Transmission dynamics of HIV, Nature, 326, pp. 137142.

[3] S. Blythe and C. Castillo-Chavez (1989), Like with like preference and sexual mixing models, Math. Biosciences, 96, pp. 221-238.

[4] S. Busenberg and C. Castillo-Chavez (1991), A general solution of the problem of mixing and its application to risk- and age-structured epidemic models for the spread of AIDS, IMA J. Math. Appl. Med. Biol., 2, pp. 1-29.

[5] S. Busenberg and C. Castillo-Chavez (1991), Interaction, pair formation, and force of infection terms in sexually transmitted diseases, in Lecture Notes in Biomath. 83, SpringerVerlag, New York, 1989. 
[6] C. Castillo-Chavez, S. Shyu, G. Rubin, and D. Umbach (1992), On the estimation problem of mixing pair formation matrices with applications to models for sexually transmitted diseases, in IMS AIDS Statistical Methodology, K. Dietz, V. T. Farewell, and N. P. Jewell, eds., IMS, Hayward, CA.

[7] C. Castillo-Chavez, S. Busenberg, and K. Gerow (1990), Pair formation in structured populations, in Differential Equations with Applications in Biology, Physics, and Engineering, J. Goldstein, F. Kappel, and W. Scheppacher, eds., Marcel Dekker, NY.

[8] C. Castillo-Chavez and S. Busenberg (1991), On the solution of the two-sex mixing problem, in Proc. International Conference on Differential Equations and Applications to Biology in Population Dynamics, S. Busenberg and M. Martelli, eds., Lecture Notes in Biomath. 92, Springer-Verlag, New York, pp. 80-98.

[9] C. Castillo-Chavez, K. Cooke, S. Huang, and S. Levin (1989), Results on the dynamics for models for the sexual transmission of HIV, Appl. Math. Lett., 4, pp. 327-331.

[10] C. Castillo-Chavez and S. Blythe (1989), Mixing framework for social/sexual behavior, in Lecture Notes in Biomath. 83, Springer-Verlag, New York.

[11] C. Castillo-Chavez (1994), On a corrected interpretation of risk-group distributions, unpublished.

[12] K. Dietz (1988), On the transmission of HIV, Math. Biosci., 90, pp. 397-414.

[13] H. Нетнсоте (1989), A dynamic model of HIV transmission and AIDS in San Francisco, presented at the Fifth International Conference on AIDS, San Francisco, Abstract, p. 149.

[14] J. M. Hyman and E. A. Stanley (1988), Using mathematical models to understand the AIDS epidemic, Math. Biosci., 90, pp. 415-473.

[15] J. M. Hyman and E. A. Stanley (1989), The effects of social mixing patterns on the spread of AIDS, in Mathematical Approaches to Problems in Resource Management and Epidemiology, C. Castillo-Chavez, S. A. Levin, and C. A. Shoemaker, eds., Lecture Notes in Biomath. 81, Springer-Verlag, New York, pp. 190-219.

[16] S. Hu-Schmitz, S. Busenerg, and C. Castillo-Chavez (1993), On the Evolution of Marriage Functions: It Takes Two to Tango, Tech. Report BU-1210-M, Cornell University, Ithaca, NY.

[17] J. M. Hyman and E. A. Stanley (1994), A risk-based heterosexual model for the AIDS epidemic with biased sexual partner selection, in Modeling the AIDS Epidemic: Policy and Prediction, E. Kaplan and F. Brandeau, eds., Raven Press, New York, pp. 511-533.

[18] J. Jacquez, C. Simon, And J. Koopman (1989), Structured mixing: Heterogeneous Mixing by the definition of activity groups, in Lecture Notes in Biomath. 83, Springer-Verlag, New York, pp. 301-315.

[19] D. E. Kirschner (1992), Examining a system of pseudo-cross diffusion equations, Internat. J. Sci. Comput. Modeling, 1, pp. 174-179.

[20] D. E. Kirschner (1996), A diffusion model for AIDS in a closed, heterosexual population: Examining rates of infection, SIAM J. Appl. Math., 56, pp. 143-166.

[21] N. Padian and J. Wiley (1987), Male to female transmission of HIV: Current results, infectivity rates and San Francisco population seroprevalence estimates, presented at III International Conference on AIDS in Washington, D.C.

[22] L. Sattenspiel (1990), Modeling the spread of infectious disease in human populations, Yearbook Phys. Anthrop., 33, pp. 22-48.

[23] E. A. Stanley (1993), Partner selection in heterosexual populations, in Mathematical Population Dynamics: Analysis of Heterogeneity, Vol. 1: Theory of Epidemics, O. Arino, M. Kimnel, M. Langlais, and D. Axelrod, eds., Wuerz, Canada, pp. 311-330.

[24] H. Thieme and C. Castillo-Chavez (1989), On the role of variable infectivity in the dynamics of the HIV epidemic, in Lecture Notes in Biomath. 83, Springer-Verlag, New York.

[25] H. R. Thieme and C. Castillo-Chavez (1993), How may infection-age-dependent infectivity affect the dynamics of HIV/AIDS?, SIAM J. Appl. Math., 53, pp. 1447-1479.

[26] J. Wiley (1990), AIDS: The second decade, Chapter 6, National Academy of Science Publication for National Research Council, San Francisco. 